\title{
A LOWER BOUND FOR FAITHFUL REPRESENTATIONS OF NILPOTENT LIE ALGEBRAS
}

\author{
LEANDRO CAGLIERO AND NADINA ROJAS
}

\begin{abstract}
In this paper we present a lower bound for the minimal dimension $\mu(\mathfrak{n})$ of a faithful representation of a finite dimensional $p$-step nilpotent Lie algebra $\mathfrak{n}$ over a field of characteristic zero. Our bound is given as the minimum of a quadratically constrained linear optimization problem, it works for arbitrary $p$ and takes into account a given filtration of $\mathfrak{n}$. We present some estimates of this minimum which leads to a very explicit lower bound for $\mu(\mathfrak{n})$ that involves the dimensions of $\mathfrak{n}$ and its center. This bound allows us to obtain $\mu(\mathfrak{n})$ for some families of nilpotent Lie algebras.
\end{abstract}

\section{INTRODUCTION AND MAIN RESULTS}

In this paper all Lie algebras and representations are finite dimensional over field k of characteristic zero. Given a representation $(\pi, V)$ of a nilpotent Lie algebra $\mathfrak{n}$, we say that $(\pi, V)$ is a nilrepresentation if $\pi(X)$ is a nilpotent for all $X \in \mathfrak{n}$.

Ado's Theorem states that any Lie algebra has a faithful representation (see [J, p. 202]). Nevertheless, given a Lie algebra $\mathfrak{n}$, the invariants

$$
\begin{aligned}
\mu(\mathfrak{n}) & =\min \{\operatorname{dim} V:(\pi, V) \text { is a faithful representation of } \mathfrak{n}\}, \\
\mu_{\text {nil }}(\mathfrak{n}) & =\min \{\operatorname{dim} V:(\pi, V) \text { is a faithful nilrepresentation of } \mathfrak{n}\} .
\end{aligned}
$$

are, in general, very difficult to compute or even to estimate. Apart from its intrinsic interest, the map $\mu$ is not only important in computational mathematics, but it is also connected to the theory of compact affine manifolds and crystallographic groups (see for instance [Be, B, K, Mi, Se2]) and to the theory of polycyclic groups (see for instance [Se1] [Ch. 5,6], GSe] [§3.2]).

The value of $\mu(\mathfrak{n})$ has been obtained only for very few families of Lie algebras $\mathfrak{n}$ (see, for instance [Be, B, BM1, CRo, $[\mathrm{Ro},[\mathrm{S}]$ ).

Obtaining general results about $\mu$, in particular new bounds, is very hard. On the one hand, there is a number of papers investigating new methods for constructing faithful representations of small dimension for a given class of (nilpotent) Lie algebras (see for instance [BM2, BEdG, dG, dGN, Ne]) and thus obtaining upper bounds for $\mu$. In this direction, an ambitious goal is to find out whether there is a fixed polynomial $p$ such that $\mu(\mathfrak{n}) \leq p(\operatorname{dim} \mathfrak{n})$ for all Lie algebras $\mathfrak{n}$ (at least inside a wide class).

2010 Mathematics Subject Classification. 17B10, 17B30, 17B35, 17B45.

Key words and phrases. Nilpotent Lie algebras, Ado's Theorem, Nilrepresentation, Minimal Faithful Representation.

Partially supported by CONICET, FONCyT and SECyT-UNC Grants (Argentina). 
On the other hand, general lower bounds are crucial for proving that a given faithful representation of a Lie algebra is actually of minimal dimension. They are also important for their applications to other problems. For instance, the counterexample obtained by Benoist [Be] to Milnor's conjecture [Mi] is based on a family of Lie algebras satisfying $\mu(\mathfrak{n})>\operatorname{dim} \mathfrak{n}+1$. On the group theory side, lower bounds for faithful representations of finite groups have been used to obtain a lower bound for the smallest non-trivial eigenvalue of the Laplace-Beltrami operator on certain manifolds [SX], or to answer questions of Lubotzky about the uniform expansion bounds for the Cayley graphs of $\mathrm{SL}_{2}\left(\mathbb{F}_{p}\right)$ [BoG].

In this paper we obtain the following lower bound of $\mu_{n i l}$ for nilpotent Lie algebras.

Theorem 1.1. Let $\mathfrak{n}$ be a Lie algebra and let $\mathfrak{n}_{p} \subset \cdots \subset \mathfrak{n}_{1}=\mathfrak{n}$ be a filtration of $\mathfrak{n}$ such that $\mathfrak{n}_{p_{0}}$ is contained in the center of $\mathfrak{n}$. Then

$$
\mu_{n i l}(\mathfrak{n}) \geq r_{0}^{\min }
$$

where $r_{0}^{\text {min }}$ is the minimum value of

$$
r_{0}=a_{0}+a_{1}+\cdots+a_{p}, \quad a_{0}, a_{1}, \ldots, a_{p} \in \mathbb{Z},
$$

subject to the following restrictions:

(a) $a_{0}, a_{p} \geq 1$ and $a_{k} \geq 0$,

for $k=1, \ldots, p-1$

(b) $\sum_{i=0}^{p_{0}-k} a_{i}\left(a_{k+i}+\cdots+a_{p}\right) \geq \operatorname{dim} \mathfrak{n}_{k}$, for $k=1, \ldots, p_{0}$;

(c) $a_{0}\left(a_{k}+\cdots+a_{p}\right) \geq \operatorname{dim} \mathfrak{n}_{k}, \quad$ for $k=p_{0}, \ldots, p$.

The quadratically constrained linear optimization problem involved in the above theorem seems to be difficult. In this paper we present some quick, but not trivial, estimations of $r_{0}^{\min }$ and the lower bounds obtained are already interesting. We are confident that future research on $r_{0}^{\mathrm{min}}$ will provide very good lower bounds for $\mu_{\text {nil }}(\mathfrak{n})$. As a consequence of our estimates, we obtain the following theorem.

Theorem 1.2. Let $\mathfrak{n}$ be a p-step nilpotent Lie algebra, $p>1$, and let $\mathfrak{z}$ be the center of $\mathfrak{n}$.

(1) If $\operatorname{dim} \mathfrak{n} \geq\left((p-1)^{2}+p^{2}\right) \operatorname{dim} \mathfrak{z}$ then

$$
\mu_{n i l}(\mathfrak{n}) \geq \sqrt{\frac{2 p}{p-1}(\operatorname{dim} \mathfrak{n}-\operatorname{dim} \mathfrak{z})} .
$$

(2) If $\operatorname{dim} \mathfrak{n} \leq\left((p-1)^{2}+p^{2}\right) \operatorname{dim} \mathfrak{z}$ then

$$
\begin{aligned}
& \quad \mu_{n i l}(\mathfrak{n}) \geq \sqrt{\frac{2(p-1)}{p-2} \operatorname{dim} \mathfrak{n}+\frac{2 p(p-1)}{(p-2)^{2}} \operatorname{dim} \mathfrak{z}}-\frac{2}{p-2} \sqrt{\operatorname{dim} \mathfrak{z}}, \\
& \text { if } p \neq 2 \text {, and } \mu_{\text {nil }}(\mathfrak{n}) \geq \frac{\operatorname{dim} \mathfrak{n}+3 \operatorname{dim} \mathfrak{z}}{2 \sqrt{\operatorname{dim} \mathfrak{z}}} \text { if } p=2 \text {. }
\end{aligned}
$$

In both cases, the given bound is bigger than $\sqrt{\frac{2(p+1)}{p} \operatorname{dim} \mathfrak{n}}$.

From this theorem, $\mu_{n i l}$ is obtained for the following families. 
(i) Given $p, a \in \mathbb{N}$, let

$$
\begin{aligned}
& \mathfrak{n}_{a, p}=\left\{\left(\begin{array}{ccccc}
0 & A_{12} & A_{13} & \ldots & A_{1 p+1} \\
0 & A_{23} & \ldots & A_{2 p+1} \\
& \ddots & & \vdots \\
0 & & & A_{p p+1}
\end{array}\right): A_{i j} \in M_{a}(\mathrm{k}) \text { para } 1 \leq i<j \leq p+1\right\} \text {, } \\
& \text { then } \mu\left(\mathfrak{n}_{a, p}\right)=(p+1) a \text {. }
\end{aligned}
$$

(ii) Given $a, b, c \in \mathbb{N}$ let

$$
\mathfrak{n}_{a, b, c}=\left\{\left(\begin{array}{ccc}
0 & A_{a b} & A_{a c} \\
0 & A_{b c} \\
& & 0
\end{array}\right): A_{a b} \in M_{a, b}(\mathrm{k}), A_{a c} \in M_{a, c}(\mathrm{k}), A_{b c}(\mathrm{k}) \in M_{b, c}\right\} .
$$

Then, if either $b=a+c$, or $a=c$ and $b \leq 2 a$,

$$
\mu\left(\mathfrak{n}_{a, b, c}\right)=a+b+c .
$$

The above two families are nilradicals of parabolic subalgebras of simple Lie algebras of type $\mathrm{A}$. The above result shows that their defining representation is faithful of minimal dimension. However this is not true for all nilradicals of type A. For instance if $a=b=1$, then the Lie algebra $\mathfrak{n}_{1,1, c}$ given in (ii) satisfies $\mu\left(\mathfrak{n}_{1,1, c}\right)=\lceil 2 \sqrt{2 c}<2+c$ for all $c \in \mathbb{N}$, as shown in [ARo].

The paper is organized as follows. In $\oint 2$ we prove Theorem 2.3 which is a key result. It allows us to obtain certain special bases for faithful representations of nilpotent Lie algebras that eventually lead, in 93 , to the optimization problem of Theorem [1.1. In this section, an open question is posed. In $\$ 4$ we compute $\mu_{n i l}$ for the families (i) and (ii). In $\$ 5$ we obtain estimates for the minimum of our optimization problem and prove Theorem 1.2 .

\section{Linearly indePEndENT SUbSETS ASSOCIATED TO CHAins OF ENDOMORPHISMS}

In this section we describe an algorithm that, given a faithful $\mathfrak{n}$-module $V$, will provide a basis of $V$ with certain special properties that will allow us to estimate $\operatorname{dim} V$.

First, we recall the following standard lemma.

Lemma 2.1. Let $V$ be a vector space and let $\mathcal{T}_{1}, \ldots, \mathcal{T}_{p}$ be vector subspaces of $\operatorname{End}(V)$. If $r_{i}=\max \left\{\operatorname{dim} \mathcal{T}_{i} v: v \in V\right\}$ and $W_{i}=\left\{w \in V: \operatorname{dim} \mathcal{T}_{i} w=r_{i}\right\}$, then $\cap_{i=1}^{p} W_{i}$ is a non-empty open dense subset of $V$. In particular, there exists $v \in V$ such that $\operatorname{dim} \mathcal{T}_{i} v=r_{i}$ for all $i=1, \ldots, p$.

Proof. Since the intersection of open dense subsets is a non-empty open dense subset, it suffices to prove that $W_{i}$ is open and dense for all $i$. Let us fix $i=1, \ldots, p$, and let $w \in W_{i}$ and let $\left\{T_{1}, \ldots, T_{r_{i}}\right\} \subseteq \mathcal{T}_{i}$ be such that

$$
\left\{T_{1}(w), \ldots, T_{r_{i}}(w)\right\}
$$

is a basis of $\mathcal{T}_{i} w$. For any $v \in V, A(v)$ denote the matrix whose columns are the coordinates of $T_{1}(v), \ldots, T_{r_{i}}(v)$ in a given basis $B$ of $V$. Since $\left\{T_{1}(w), \ldots, T_{r}(w)\right\}$ is a linearly independent set, the matrix $A(v)$ has an $(r \times r)$-minor $a(v)$ such that $\operatorname{det} a(w) \neq 0$. Therefore, the open set $U=\{v \in$ $V: \operatorname{det} a(v) \neq 0\}$ contains $w$, is contained in $W_{i}$ and, since $\mathrm{k}$ is an infinite field, it is dense. 
Definition 2.2. Given a vector space $V$ and a sequence of vector subspaces $\mathcal{T}_{1}, \ldots, \mathcal{T}_{p}$ of $\operatorname{End}(V)$, we say that $v \in V$ is rank-vector for the sequence $\mathcal{T}_{1}, \ldots, \mathcal{T}_{p}$, if

$$
\operatorname{dim} \mathcal{T}_{i} v=\max \left\{\operatorname{dim} \mathcal{T}_{i} w: w \in V\right\}
$$

for all $i=1, \ldots, p$.

Let $\mathcal{T}_{p} \subset \cdots \subset \mathcal{T}_{1}=\mathcal{T}$ be a chain of vector subspaces of $\operatorname{End}(V)$ and let $\left\{v_{1}, v_{2}, v_{3}, \ldots\right\}$ the sequence (which eventually will be finite) obtained by applying the following procedure:

(1) choose a rank-vector $v_{1}$ for the chain $\mathcal{T}$,

(2) choose a (special) linear complement $\mathcal{T}^{\prime}$ of the annihilator of $v_{1}$ in $\mathcal{T}$,

(3) choose a rank-vector $v_{2}$ for the chain $\mathcal{T}^{\prime}$,

and so on. More precisely, the procedure is given by the following algorithm.

(i) For all $k=1, \ldots, p$, let $s_{k}:=0$ and $\mathcal{R}_{k}:=\mathcal{T}_{k}$.

Let $i:=0, q:=p$.

(ii) Increase $i$ by 1 .

(iii) Let $v_{i}$ be a rank-vector associated to $\mathcal{R}_{q} \subset \cdots \subset \mathcal{R}_{1}$.

(iv) For all $k=1, \ldots, q$, let

$$
\tilde{\mathcal{R}}_{k}=\operatorname{Ann}_{\mathcal{R}_{k}}\left(v_{i}\right)=\left\{T \in \mathcal{R}_{k}: T\left(v_{i}\right)=0\right\},
$$

If $\tilde{\mathcal{R}}_{k} \neq \mathcal{R}_{k}$, increase $s_{k}$ by 1 and let $\mathcal{T}_{k, i}$ be such that

$$
\mathcal{R}_{k}=\mathcal{T}_{k, i} \oplus \tilde{\mathcal{R}}_{k} \text { and } \mathcal{T}_{k, i} \supseteq \mathcal{T}_{k+1, i} \quad \text { (assume } \mathcal{T}_{q+1, i}=0 \text { ). }
$$

(v) If $\tilde{\mathcal{R}}_{1} \neq 0$, let $q$ be the largest $j$ such that $\tilde{\mathcal{R}}_{j} \neq 0$ and let

$$
\mathcal{R}_{k}:=\tilde{\mathcal{R}}_{k}, \quad k=1, \ldots, q,
$$

(we have $\mathcal{R}_{q} \subset \cdots \subset \mathcal{R}_{1}$ ). Go to (ii).

(vi) End.

As a result we obtain:

(a) A partition $s_{1} \geq s_{2} \geq \cdots \geq s_{p}>0$, ( $s_{1}$ is the final value of $\left.i\right)$.

(b) A set $\left\{v_{1}, v_{2}, \ldots, v_{s_{1}}\right\}$.

(c) A family of subspaces $\mathcal{T}_{k, j} \subset \operatorname{End}(V), 1 \leq j \leq s_{k}$ and $1 \leq k \leq p$.

The following theorem summarizes some of the main properties of the set $\left\{v_{1}, v_{2}, \ldots, v_{s_{1}}\right\}$ and the family of subspaces $\mathcal{T}_{k, j} \subset \operatorname{End}(V)$.

Theorem 2.3. Let $V$ be a vector space and let $\mathcal{T}_{p} \subset \cdots \subset \mathcal{T}_{1}$ be a chain of subspaces in $\operatorname{End}(V)$. Then there exist a partition $s_{1} \geq s_{2} \geq \cdots \geq s_{p}>0$, a linearly independent set $\left\{v_{1}, \ldots, v_{s_{1}}\right\} \subset V$ and a family of subspaces $\mathcal{T}_{k, j} \subset$ $\operatorname{End}(V), 1 \leq j \leq s_{k}$ and $1 \leq k \leq p$, such that: 


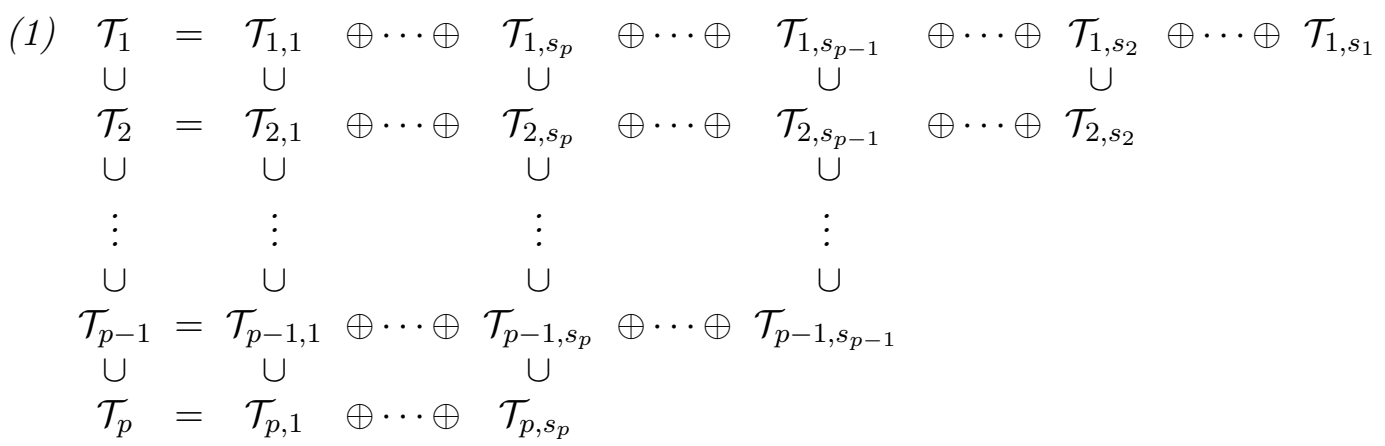

We notice that this display resembles the Young diagram of the partition $s_{1} \geq s_{2} \geq \cdots \geq s_{p}$.

(2) $\operatorname{dim} \mathcal{T}_{k, j}=\operatorname{dim} \mathcal{T}_{k, j} v_{j}$ for $j=1, \ldots, s_{k}$ and $k=1, \ldots, p$.

(3) $\mathcal{T}_{k, j} v_{i}=0$ for $1 \leq i<j \leq s_{k}$ and $k=1, \ldots, p$.

(4) $\mathcal{T}_{k, j} V \subseteq \mathcal{T}_{k, i} v_{i}$ for $1 \leq i<j \leq s_{k}$ and $k=1, \ldots, p$.

Moreover, if $\mathcal{T}_{1}$ consists of nilpotent operators and $\left[\mathcal{T}_{1}, \mathcal{T}_{p_{0}}\right]=0,1 \leq p_{0} \leq p$, then $\mathcal{T}_{1,1} v_{1} \cap \operatorname{span}_{\mathrm{k}}\left\{v_{1}, \ldots, v_{s_{p_{0}}}\right\}=0$.

Proof. By construction, it is clear that properties (1), (2) and (3) hold.

We first prove that $\left\{v_{1}, v_{2}, \ldots, v_{s_{1}}\right\}$ is linearly independent. By construction, we may assume, as an induction hypothesis, that $\left\{v_{2}, \ldots, v_{s_{1}}\right\}$ is linearly independent. Thus we must show that $v_{1} \notin \operatorname{span}_{\mathrm{k}}\left\{v_{2}, \ldots, v_{s_{1}}\right\}$.

If

$$
v_{1}=\sum_{j=2}^{s_{1}} a_{j} v_{j}
$$

let $j_{0}=\max \left\{j: a_{j} \neq 0\right\} \geq 2$ and let $T \in \mathcal{T}_{1, j_{0}} \subset \mathcal{T}_{1}, T \neq 0$. We now apply $T$ to both sides of (2.1). Property (3) implies that the left-hand side is zero and the right hand side is $a_{j_{0}} T\left(v_{j_{0}}\right)$. On the other hand, property (2) says that $T\left(v_{j_{0}}\right) \neq 0$, which is a contradiction.

We now prove (4). If $s_{1}=1$ then $\mathcal{T}_{k, 1}=\mathcal{T}_{k}$ for all $k=1, \ldots, p$ and condition (4) is empty. As we did earlier, we may assume by induction that $\mathcal{T}_{k, j} V \subseteq \mathcal{T}_{k, i} v_{i}$ for $2 \leq i<j \leq s_{k}$ and $k=1, \ldots, p$. Thus, we only need to prove (4) when $i=1$. This is equivalent to prove that $T(v) \in \mathcal{T}_{k, 1} v_{1}$ for all $v \in V$ and all $T \in \mathcal{T}_{k, 2} \oplus \cdots \oplus \mathcal{T}_{k, s_{k}}, k=1, \ldots, p$. If $r_{k}=\operatorname{dim} \mathcal{T}_{k, 1} v_{1}$ and $\left\{T_{1}, \ldots, T_{r_{k}}\right\}$ is a basis of $\mathcal{T}_{k, 1}$, we must show that

$$
\left\{T(v), T_{1}\left(v_{1}\right), \ldots, T_{r_{k}}\left(v_{1}\right)\right\}
$$

is linearly dependent for all $T \in \mathcal{T}_{k, 2} \oplus \cdots \oplus \mathcal{T}_{k, s_{k}}, k=1, \ldots, p$, and all $v \in V$.

Let us fix such $T, v$ and $k$. By the definition of $v_{1}$, the set

$$
\left\{T\left(v_{1}+t v\right), T_{1}\left(v_{1}+t v\right), \ldots, T_{r_{k}}\left(v_{1}+t v\right)\right\}
$$

is linearly dependent for all $t \in \mathrm{k}$. Since $T\left(v_{1}\right)=0$, we obtain that $\left\{T(v), T_{1}\left(v_{1}+t v\right), \ldots, T_{r_{k}}\left(v_{1}+t v\right)\right\}$ is linearly dependent for all $t \neq 0$. Since $\mathrm{k}$ infinite, we conclude that this last set is linearly dependent for $t=0$. This completes the proof of (4).

We now prove the 'moreover' part of the theorem. We must show that

$$
\mathcal{T}_{1,1} v_{1} \cap \operatorname{span}_{\mathrm{k}}\left\{v_{1}, \ldots, v_{s_{p_{0}}}\right\}=0 .
$$


Suppose, on the contrary, that there exist $T \in \mathcal{T}_{1,1}, T \neq 0$, and $a_{1}, \ldots, a_{s_{p_{0}}} \in$ $\mathrm{k}$ such that

$$
T\left(v_{1}\right)=\sum_{j=0}^{s_{p_{0}}} a_{j} v_{j} .
$$

Since $T \in \mathcal{T}_{1,1}$ and $T \neq 0$, it follows that $T\left(v_{1}\right) \neq 0$ and thus $a_{j} \neq 0$ for some $j$. Let $j_{0}=\max \left\{j: a_{j} \neq 0\right\}$. Since $T$ is nilpotent, its only eigenvalue is zero and thus $1<j_{0} \leq s_{p_{0}}$.

Let $T^{\prime} \in \mathcal{T}_{p_{0}, j_{0}}, T^{\prime} \neq 0$, and let us apply $T^{\prime}$ to both sides of (2.2). Since $T^{\prime} \in \mathcal{T}_{p_{0}, j_{0}}$ and $j_{0}>1$ we obtain on the left hand side $T^{\prime} T\left(v_{1}\right)=T T^{\prime}\left(v_{1}\right)=0$. On the other hand, it follows from properties (2) and (3) that the right hand side is $a_{j_{0}} T^{\prime}\left(v_{j_{0}}\right) \neq 0$, which is a contradiction.

\section{An Optimization PROBlem LEADING TO A LOWER BOUND FOR $\mu_{\text {nil }}$}

Let $V$ be a vector space and let $\mathfrak{n}$ be a Lie subalgebra of $\mathfrak{g l}(V)$ consisting of nilpotent endomorphisms. Let

$$
\mathfrak{n}_{p} \subset \cdots \subset \mathfrak{n}_{2} \subset \mathfrak{n}_{1}=\mathfrak{n}
$$

be a filtration of $\mathfrak{n}$ such that $\mathfrak{n}_{p_{0}}$ is contained in the center of $\mathfrak{n}$ for some $1 \leq p_{0} \leq p$.

Applying Theorem 2.3 to the filtration $\mathfrak{n}_{p} \subset \cdots \subset \mathfrak{n}_{2} \subset \mathfrak{n}_{1}=\mathfrak{n}$ we obtain a partition $s_{1} \geq s_{2} \geq \cdots \geq s_{p}>0$, a linearly independent set $\left\{v_{1}, \ldots, v_{s_{1}}\right\} \subset V$, and a decomposition

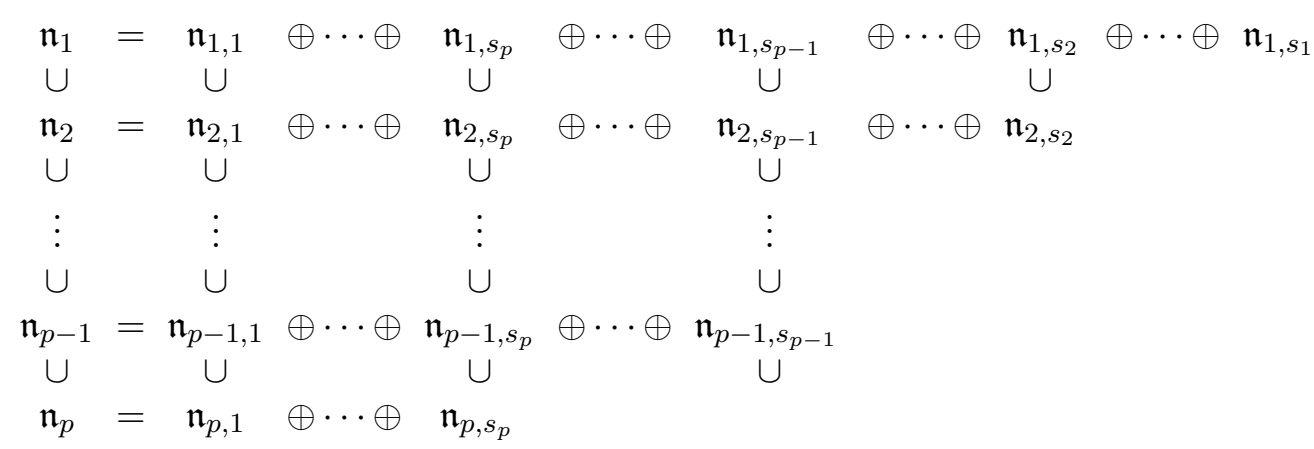

such that

$$
\mathfrak{n}_{1,1} v_{1} \cap \operatorname{span}_{\mathrm{k}}\left\{v_{1}, \ldots, v_{s_{p_{0}}}\right\}=0 .
$$

Let $r_{k}=\operatorname{dim} \mathfrak{n}_{k, 1}$. Since $\mathfrak{n}_{k, 1} \subseteq \mathfrak{n}_{k-1,1}$, there exists a basis $\left\{X_{1}, \ldots, X_{r_{1}}\right\}$ of $\mathfrak{n}_{1,1}$ such that $\left\{X_{1}, \ldots, X_{r_{k}}\right\}$ is a basis of $\mathfrak{n}_{k, 1}, k=1, \ldots, p$.

It follows from Theorem 2.3 that

$$
\left\{X_{1}\left(v_{1}\right), \ldots, X_{r_{k}}\left(v_{1}\right)\right\}
$$

is a basis of $\mathfrak{n}_{k, 1} v_{1}, k=1, \ldots, p$. We now fix an ordered basis

$$
B=\left\{X_{1}\left(v_{1}\right), \ldots, X_{r_{1}}\left(v_{1}\right), w_{1}, \ldots, w_{q}, v_{1}, \ldots, v_{s_{p_{0}}}\right\}
$$

of $V$ and let

$$
\begin{aligned}
& W=\operatorname{span}_{\mathrm{k}}\left\{w_{1}, \ldots, w_{q}\right\}, \\
& V_{0}=\operatorname{span}_{\mathrm{k}}\left\{v_{1}, \ldots, v_{s_{p_{0}}}\right\} .
\end{aligned}
$$


We now consider the matrix of a given $X \in \mathfrak{n}$ with respect to the basis $B$

$$
\left.\left.[X]_{B}=\left[\begin{array}{lll}
\overbrace{A_{1,1}(X)}^{r_{1}} & \overbrace{A_{1,2}(X)}^{q} & \overbrace{A_{1,3}(X)}^{s_{p_{0}}} \\
A_{2,1}(X) & A_{2,2}(X) & A_{2,3}(X) \\
A_{3,1}(X) & A_{3,2}(X) & A_{3,3}(X)
\end{array}\right]\right\} r_{r_{1}}\right\}
$$

where the row and columns correspond to the decomposition

$$
V=\mathfrak{n}_{1,1} v_{1} \oplus W \oplus V_{0} .
$$

The following proposition describe the main properties of $[X]_{B}$.

Proposition 3.1. Let $X \in \mathfrak{n}_{k, j}$ for some $k=1, \ldots, p$ and $j=1, \ldots, s_{k}$.

(1) If $j=1$ then $\left(A_{1,3}(X)\right)_{h, 1}=0$ for all $h=r_{k}+1, \ldots, r_{1}$. In addition, $\left(A_{1,3}(X)\right)_{h, 1}=0$ for all $h=1, \ldots, r_{1}$ if and only if $X=0$.

(2) If $j \geq 2$ then $A_{m, n}(X)=0$ for $m=2,3, n=1,2,3$. On the other hand, the row $A_{1,1}(X) A_{1,2}(X) A_{1,3}(X)$ has the following structure

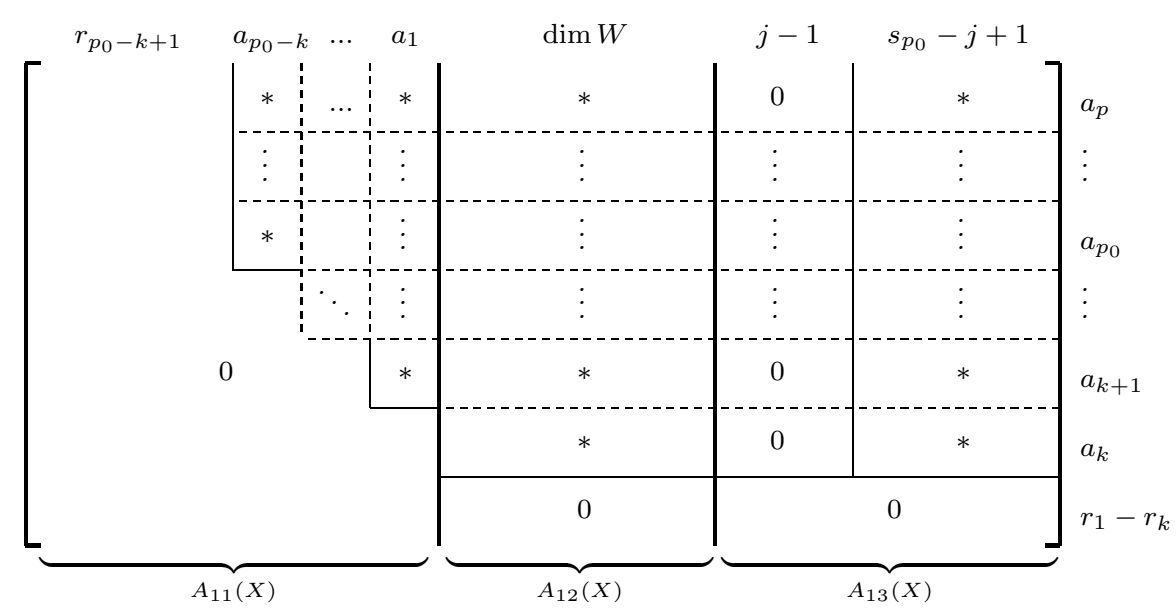

where $a_{h}=r_{h}-r_{h+1}, h=1, \ldots, p-1$ and $a_{p}=r_{p}$. In particular, if $k \geq p_{0}$, then $A_{1,1}(X)=0$.

Proof. Part (1) is a consequence of Theorem 2.3(1) and (4).

If $j \geq 2$, it follows from Theorem 2.3(4) that $X(v) \in \mathfrak{n}_{k, 1} v_{1}$ for all $v \in V$. This proves that $\left(A_{1, *}(X)\right)_{h, *}=0$ for all $h \geq k$. It follows from Theorem 2.3(3) that $\left(A_{1,3}(X)\right)_{*, h}=0$ for all $h \leq j-1$.

Finally, let us prove that $A_{1,1}(X)$ has the staircase-shape stated above. If $i=1, \ldots, r_{1}$, then $i^{\text {th }}$ element of $B$ is $X_{i}\left(v_{1}\right)$. If additionally $i \leq r_{h}$, for some $h=1, \ldots, p$, then $X_{i} \in \mathfrak{n}_{h, 1}$ and since $X \in \mathfrak{n}_{k, j}$ we obtain $\left[X, X_{i}\right] \in \mathfrak{n}_{k+h}$. Thus

$$
\begin{aligned}
X X_{i}\left(v_{1}\right) & =X_{i} X\left(v_{1}\right)+\left[X, X_{i}\right]\left(v_{1}\right) \\
& =\left[X, X_{i}\right]\left(v_{1}\right) \in \mathfrak{n}_{k+h} v_{1} \quad(\text { since } j \geq 2) .
\end{aligned}
$$

This completes the proof. 
Question. Since $\mathfrak{n} \subset \mathfrak{g l}(V)$ consists of nilpotent endomorphisms, it would be very interesting to obtain a basis $B$ such that $[X]_{B}$ is upper triangular for all $X \in \mathfrak{n}$, in addition to the properties stated in Proposition 3.1 (or similar ones). This would transform Proposition 3.1 into a detailed version of Lie's Theorem that takes into account a given filtration of the Lie algebra $\mathfrak{n}$. As stated, Proposition 3.1 is enough to obtain the lower bounds that we are looking for.

Theorem 3.2. Let $\mathfrak{n}$ be a Lie subalgebra of nilpotent operators of $\mathfrak{g l}(V)$ and let $\mathfrak{n}_{p} \subset \cdots \subset \mathfrak{n}_{1}=\mathfrak{n}$ be a filtration of $\mathfrak{n}$ such that $\mathfrak{n}_{p_{0}}$ is contained in the center of $\mathfrak{n}$. Then there exists integers $a_{k} \geq 0, k=0, \ldots, p$, with $a_{0}, a_{p} \geq 1$, such that:

(1) $\operatorname{dim} \mathfrak{n}_{k} \leq \sum_{i=0}^{p_{0}-k} a_{i}\left(a_{k+i}+\cdots+a_{p}\right)$ for $k=1, \ldots, p_{0}$.

(2) $\operatorname{dim} \mathfrak{n}_{k} \leq a_{0}\left(a_{k}+\cdots+a_{p}\right)$ for $k=p_{0}, \ldots, p$.

(3) $\operatorname{dim} V=a_{0}+a_{1}+\cdots+a_{p}$.

Proof. Let $B$ be the basis of $V$ as in (3.3) and let $T: \mathfrak{n} \rightarrow \mathfrak{g l}(V) \oplus V$ be defined by

$$
T(X)= \begin{cases}X\left(v_{1}\right) \in V, & \text { if } X \in \mathfrak{n}_{1,1} \\ X \in \mathfrak{g l}(V), & \text { if } X \in \mathfrak{n}_{1, j}, j \geq 2 .\end{cases}
$$

It follows from Theorem 2.3 that $T$ is injective. We apply Proposition 3.1 to obtain a bound for $\operatorname{dim} T\left(\mathfrak{n}_{k}\right)$. On the one hand, we know from Theorem $2.3(2)$ that

$$
\operatorname{dim} T\left(\mathfrak{n}_{k, 1}\right)=r_{k} .
$$

On the other hand, from Proposition 3.1 (2), when $j \geq 2$, we know the shape of the matrices $\left[T\left(\mathfrak{n}_{k, j}\right)\right]_{B}$. Taking into account that the first column of $A_{1,3}\left(T\left(\mathfrak{n}_{k, j}\right)\right)$ is zero if $j \geq 2$, we obtain

$$
\operatorname{dim} T\left(\bigoplus_{j=2}^{s_{k}} n_{k, j}\right) \leq \underbrace{\left(\operatorname{dim} W+\mathfrak{s}_{p_{0}}\right) r_{k}-r_{k}}_{\begin{array}{c}
\text { size of } A_{1,2} \text { and } A_{1,3} \\
\text { except the } 1^{\text {st }} \text { column of } A_{1,3}
\end{array}}+\underbrace{a_{1} r_{k+1}+a_{2} r_{k+2}+\cdots+a_{p_{0}-k} r_{p_{0}}}_{\begin{array}{c}
\text { size of the staircase in } A_{1,1}, \\
\text { it appears only if } k<p_{0}
\end{array}}
$$

where $a_{h}=r_{h}-r_{h+1} \geq 0, h=1, \ldots, p-1$. Therefore

$$
\operatorname{dim} T\left(n_{k}\right) \leq \begin{cases}\left(\operatorname{dim} W+\mathfrak{s}_{p_{0}}\right) r_{k}+\sum_{i=1}^{p_{0}-k} a_{i} r_{k+i}, & \text { if } k<p_{0} \\ \left(\operatorname{dim} W+\mathfrak{s}_{p_{0}}\right) r_{k}, & \text { if } k \geq p_{0} .\end{cases}
$$

If $a_{p}=r_{p} \geq 1$ and $a_{0}=\operatorname{dim} W+\mathfrak{s}_{p_{0}} \geq 1$, then rewriting the above inequality in terms of $a_{k}{ }^{\prime} s$, we obtain

$$
\operatorname{dim} T\left(n_{k}\right) \leq \begin{cases}a_{0}\left(a_{k}+\cdots+a_{p}\right)+\sum_{i=1}^{p_{0}-k} a_{i}\left(a_{k+i}+\cdots+a_{p}\right), & \text { if } k<p_{0} \\ a_{0}\left(a_{k}+\cdots+a_{p}\right), & \text { if } k \geq p_{0} .\end{cases}
$$

This shows (1) and (2).

Finally $a_{0}+a_{1}+\cdots+a_{p}=\operatorname{dim} W+\mathfrak{s}_{p_{0}}+r_{1}=\operatorname{dim} V$. 
Theorem 3.2 leads us to consider the following optimization problem.

Problem 3.3. Given integer numbers $p \geq p_{0} \geq 1$ and $n_{1}, \ldots, n_{p}$, let

$$
r_{k}=a_{k}+a_{k+1}+\cdots+a_{p}, \quad a_{0}, a_{1}, \ldots, a_{p} \in \mathbb{Z},
$$

for $k=0, \ldots, p$. Find the minimum value $r_{0}^{\min }$ of

$$
r_{0}=a_{0}+a_{1}+\cdots+a_{p},
$$

subject to the following restrictions

(a) $a_{0}, a_{p} \geq 1$ and $a_{k} \geq 0$, for $k=1, \ldots, p-1$;

(b) $\sum_{i=0}^{p_{0}-k} a_{i} r_{k+i} \geq n_{k}, \quad$ for $k=1, \ldots, p_{0}$;

(c) $a_{0} r_{k} \geq n_{k}, \quad$ for $k=p_{0}, \ldots, p$.

The solution to this problem gives us a lower bound for $\mu_{\text {nil }}$.

Corollary 3.4. Let $\mathfrak{n}$ be a Lie algebra and let $\mathfrak{n}_{p} \subset \cdots \subset \mathfrak{n}_{1}=\mathfrak{n}$ be a filtration of $\mathfrak{n}$ such that $\mathfrak{n}_{p_{0}}$ is contained in the center of $\mathfrak{n}$. Then

$$
\mu_{n i l}(\mathfrak{n}) \geq r_{0}^{\min }
$$

where $r_{0}^{\text {min }}$ is the minimum value of Problem 3.3 associated to $p \geq p_{0} \geq 1$ and $n_{k}=\operatorname{dim} \mathfrak{n}_{k}$ with $k=1, \ldots, p$.

The optimization problem above seems to be difficult and, in this paper, we will just give a pair of quick, but not trivial, estimates of its solution. In the last section we discuss two simplifications of Problem 3.3 that lead respectively to Theorem 3.5 and Theorem 3.6 below. We think that it is worth studying Problem 3.3 in more detail in the future to obtain more accurate results than the following two theorems.

Theorem 3.5 (First simplification). Let $\mathfrak{n}$ be a nilpotent Lie algebra and let $\mathfrak{n}_{p} \subset \cdots \subset \mathfrak{n}_{1}=\mathfrak{n}$ be a filtration of $\mathfrak{n}$ such that $\mathfrak{n}_{p_{0}}$ is contained in the center of $\mathfrak{n}$ for some $p_{0}=1, \ldots, p$. Then

$$
\mu_{\text {nil }}(\mathfrak{n}) \geq \sqrt{\frac{2\left(p_{0}+1\right)}{p_{0}} \operatorname{dim} \mathfrak{n} .}
$$

In particular, if $\mathfrak{n}$ is $p$-step nilpotent Lie algebra then $\mu_{\text {nil }}(\mathfrak{n}) \geq \sqrt{\frac{2(p+1)}{p} \operatorname{dim} \mathfrak{n}}$.

Theorem 3.6 (Second simplification). Let $\mathfrak{n}$ be a nilpotent Lie algebra and let $\mathfrak{n}_{p} \subset \cdots \subset \mathfrak{n}_{1}=\mathfrak{n}(p>1)$ be a filtration of $\mathfrak{n}$ such that $\mathfrak{n}_{p_{0}}$ is contained in the center of $\mathfrak{n}$ for some $p_{0}=2, \ldots, p$ and let $n_{i}=\operatorname{dim} \mathfrak{n}_{i}, i=1, \ldots, p$.

(1) If $n_{1} \geq\left(\left(p_{0}-1\right)^{2}+p_{0}^{2}\right) n_{p_{0}}$ then

$$
\mu_{n i l}(\mathfrak{n}) \geq \sqrt{\frac{2 p_{0}}{p_{0}-1}\left(n_{1}-n_{p_{0}}\right)} .
$$


(2) If $n_{1} \leq\left(\left(p_{0}-1\right)^{2}+p_{0}^{2}\right) n_{p_{0}}$ then

$$
\mu_{n i l}(\mathfrak{n}) \geq \sqrt{\frac{2\left(p_{0}-1\right)}{p_{0}-2} n_{1}+\frac{2 p_{0}\left(p_{0}-1\right)}{\left(p_{0}-2\right)^{2}} n_{p_{0}}}-\frac{2}{p_{0}-2} \sqrt{n_{p_{0}}},
$$

if $p_{0} \neq 2$, and $\mu_{n i l}(\mathfrak{n}) \geq \frac{n_{1}+3 n_{2}}{2 \sqrt{n_{2}}}$, if $p_{0}=2$.

In both cases, the given bound is bigger than $\sqrt{\frac{2\left(p_{0}+1\right)}{p_{0}} n_{1}}$.

Both results are proved in $\$ 5$. Although Theorem 1.2 is an immediate corollary of Theorem 3.6 they will be treated separately since it is much easier to obtain directly Theorem 1.2 from Corollary 3.4. This will also show some of the difficulties involved in Problem 3.3 .

\section{Some APplications}

(1) Given $p, a \in \mathbb{N}$, let

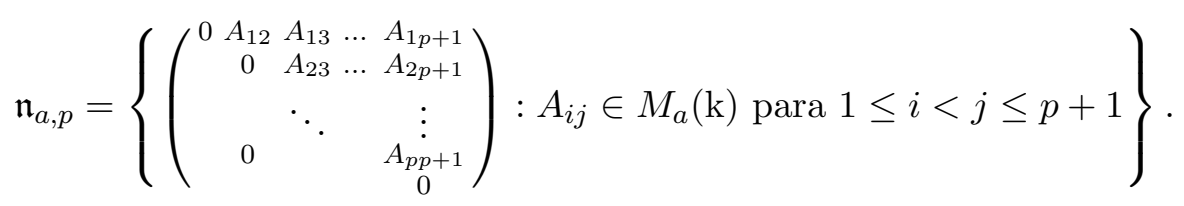

It is clear that $\mathfrak{n}_{a, p}$ is a $p$-step nilpotent Lie subalgebra of $\mathfrak{s l}((p+1) a, \mathrm{k})$ and $\operatorname{dim} \mathfrak{n}_{a, p}=\frac{(p+1) p}{2} a^{2}$. Its defining representation has dimension $(p+$ 1)a. Since Theorem 3.5 states that

$$
\mu\left(\mathfrak{n}_{a, p}\right) \geq \sqrt{\frac{2(p+1)}{p} \operatorname{dim} \mathfrak{n}_{a, p}}=(p+1) a,
$$

we obtain $\mu\left(\mathfrak{n}_{a, p}\right)=(p+1) a$.

(2) Given $a, b, c \in \mathbb{N}$ let

$$
\mathfrak{n}_{a, b, c}=\left\{\left(\begin{array}{ccc}
0 & A_{a b} & A_{a c} \\
0 & A_{b c} \\
& & 0
\end{array}\right): A_{a b} \in M_{a, b}(\mathrm{k}), A_{a c} \in M_{a, c}(\mathrm{k}), A_{b c}(\mathrm{k}) \in M_{b, c}\right\} .
$$

Now $\mathfrak{n}_{a, b, c}$ is a 2-step nilpotent Lie subalgebra of $\mathfrak{s l}(a+b+c, \mathrm{k})$ and $\operatorname{dim} \mathfrak{n}_{a, p}=a b+b c+a c$. The center of $\mathfrak{n}_{a, b, c}$ is the dimension $a c$.

If $b=a+c$ then we are under the conditions stated in part (1), Theorem 1.2 and the given lower bound for $\mu\left(\mathfrak{n}_{a, b, c}\right)$ coincides with the dimension of the defining representation of $\mathfrak{n}_{a, b, c}$.

If $a=c$ and $b \leq 2 a$ then we are under the conditions stated in (2), Theorem 1.2 and the given lower bound for $\mu\left(\mathfrak{n}_{a, b, c}\right)$ coincides with the dimension of the defining representation of $\mathfrak{n}_{a, b, c}$.

Thus, if either $b=a+c$, or $a=c$ and $b \leq 2 a$, we have

$$
\mu\left(\mathfrak{n}_{a, b, c}\right)=a+b+c .
$$

We point out that in some cases $\mu\left(\mathfrak{n}_{a, b, c}\right)<a+b+c$. For instance, it is shown in [ARO] that

$$
\mu\left(\mathfrak{n}_{1,1, c}\right)=\lceil 2 \sqrt{2 c}\rceil<1+1+c .
$$

for all $c \in \mathbb{N}$. 


\section{Estimates for the solution of Problem 3.3}

In this section we will show some bounds for $r_{0}^{\min }$ resulting from considering Problem 3.3 with real (instead of integer) variables. Since $r_{0}$ is linear, it is clear that, in this case, $r_{0}^{\min }$ will be reached in a boundary point of the restriction set.

5.1. A first simplification. It is clar that $r_{0}^{\min }$ is greater than or equal to the minimum of

$$
r_{0}=a_{0}+\cdots+a_{p}
$$

subject to

(a') $a_{0}, a_{p}>0$ and $a_{k} \in \mathbb{R}_{\geq 0}$, for $k=0, \ldots, p$;

(b') $\sum_{i=0}^{p_{0}-1} a_{i} r_{1+i} \geq n_{1}$;

We will think $r_{p_{0}}$ as an independent variable in this problem and thus we can reformulate it looking for a minimum of

$$
r_{0}=a_{0}+\cdots+a_{p_{0}-1}+r_{p_{0}}
$$

subject to

(a') $a_{0}, r_{p_{0}}>0$ and $a_{k} \in \mathbb{R}_{\geq 0}$, for $k=0, \ldots, p_{0}-1$;

$$
\text { (b') } \sum_{i=0}^{p_{0}-1} a_{i} r_{1+i} \geq n_{1} ; \quad \text { (here } r_{i}=a_{i}+\cdots+a_{p_{0}-1}+r_{p_{0}} \text { ) }
$$

Let us call this problem as Problem (a'b') for $p_{0}$. We notice that, if we consider Problem (a'b') for $p_{0}$ with the additional restriction $a_{k}=0$ for some $k=1, \ldots, p_{0}-1$, then the problem becomes Problem (a'b') for $p_{0}-1$. Therefore, in order to find the minimum value of $r_{0}$ we may consider $a_{k}>0$ for all $k=0, \ldots, p_{0}-1$. Moreover, since the minimum will be reached at the boundary, we can reformulate Problem (a'b') for $p_{0}$ as: find the minimum of $r_{0}$ subject to

(a') $a_{k}, r_{p_{0}}>0, \quad$ for $k=0, \ldots, p_{0}-1$;

(b') $\sum_{i=0}^{p_{0}-1} a_{i} r_{1+i}=n_{1}$.

We now will find the minimum value of $r_{0}$ in this problem. We use (b') in order to eliminate the variable $a_{0}$. Thus we will think $r_{0}$ as a function of $a_{1}, \ldots, a_{p_{0}-1}, r_{p_{0}}$, and we will find the critical values of $r_{0}$, and next its minimum.

It is not difficult to see that the only critical value of $r_{0}$ is

$$
\left(a_{1}, \ldots, a_{p_{0}-1}, r_{p_{0}}\right)=\left(a_{0}, a_{0}, \ldots, a_{0}, a_{0}\right)
$$

with $n_{1}=\frac{p_{0}\left(p_{0}+1\right)}{2} a_{0}^{2}$. Also, it is not difficult to see that this is a local minimum and it yields

$$
r_{0}=\sqrt{\frac{2\left(p_{0}+1\right)}{p_{0}} n_{1}}
$$


This is in fact a global minimum. Indeed, since $\sqrt{\frac{2\left(p_{0}+1\right)}{p_{0}} n_{1}}$ is decreasing as a function of $p_{0}$, taking into account the remark explained above, we can not obtain smaller values of $r_{0}$ by allowing $a_{k}=0$ for some $k$.

5.2. A second simplification. In this case, we can do an analysis similar to what we did in the first simplification to conclude that $r_{0}^{\min }$ is greater than or equal to the minimum of

$$
r_{0}=a_{0}+\cdots+a_{p_{0}-1}+r_{p_{0}}
$$

subject to

$$
\begin{aligned}
& \text { (a') } a_{k}, r_{p_{0}}>0, \quad \text { for } k=0, \ldots, p_{0}-1 \text {; } \\
& \text { (b') } \sum_{i=0}^{p_{0}-1} a_{i} r_{1+i}=n_{1} ; \\
& \text { (c') } a_{0} r_{p_{0}}=n_{p_{0}},
\end{aligned}
$$

In this case we will use (b') and (c') in order to eliminate the variables $r_{p_{0}}$ and $a_{p_{0}-1}$. Thus we will think $r_{0}$ as a function of $a_{0}, \ldots, a_{p_{0}-2}$, we will find its critical values, and its minimum.

It follows from $\left(c^{\prime}\right)$ that

$$
\frac{\partial r_{p_{0}}}{\partial a_{j}}=\left\{\begin{array}{ll}
-\frac{r_{p_{0}}}{a_{0}}, & j=0 ; \\
0, & 1 \leq j \leq p_{0}-2 .
\end{array} \quad, \quad \frac{\partial^{2} r_{p_{0}}}{\partial a_{i} a_{j}}= \begin{cases}\frac{2 r_{p_{0}}}{a_{0}^{2}}, & i=j=0 \\
0, & 1 \leq i, j \leq p_{0}-2 .\end{cases}\right.
$$

It follows from (b') that

$$
n_{1}=\left(r_{0}-r_{p_{0}-1}\right)\left(a_{p_{0}-1}+r_{p_{0}}\right)+a_{p_{0}-1} r_{p_{0}}+\sum_{i=0}^{p_{0}-3} a_{i}\left(r_{i+1}-r_{p_{0}-1}\right),
$$

and we obtain from $(\underline{5.3})$

$$
\frac{\partial a_{p_{0}-1}}{\partial a_{j}}= \begin{cases}-\frac{\left(r_{0}-a_{0}-r_{p_{0}}\right)\left(a_{0}-r_{p_{0}}\right)}{\left(r_{0}-a_{p_{0}-1}\right) a_{0}}, & j=0 \\ -\frac{r_{0}-a_{j}}{r_{0}-a_{p_{0}-1}}, & 1 \leq j \leq p_{0}-2 .\end{cases}
$$

and

$$
\frac{\partial^{2} a_{p_{0}-1}}{\partial a_{i} a_{j}}= \begin{cases}\frac{2\left(r_{0}-a_{0}-r_{p_{0}}\right)\left(a_{0}^{2}-r_{p_{0}}\left(2 a_{0}+r_{0}-r_{p_{0}-1}\right)\right)}{\left(r_{0}-a_{p_{0}-1}\right)^{2} a_{0}^{2}}, & i=j=0 ; \\ \frac{\left(r_{0}+a_{p_{0}-1}-a_{0}-a_{i}-r_{p_{0}}\right)\left(a_{0}-r_{p_{0}}\right)}{\left(r_{0}-a_{p_{0}-1}\right)^{2} a_{0}}, & 0=j<i \leq p_{0}-2 ; \\ \frac{\left(r_{0}+a_{p_{0}-1}-a_{j}-a_{i}\right)}{\left(r_{0}-a_{p_{0}-1}\right)^{2}}, & 0<j<i \leq p_{0}-2 ; \\ \frac{2\left(r_{0}-a_{j}\right)}{\left(r_{0}-a_{p_{0}-1}\right)^{2}}, & 0<j=i \leq p_{0}-2 ;\end{cases}
$$


Therefore, it follows from (5.1), (5.2) and (5.4) that

$$
\frac{\partial r_{0}}{\partial a_{j}}= \begin{cases}\frac{\left(r_{p_{0}}+a_{0}-a_{p_{0}-1}\right)\left(a_{0}-r_{p_{0}}\right)}{\left(r_{0}-a_{p_{0}-1}\right) a_{0}}, & j=0 ; \\ \frac{a_{j}-a_{p_{0}-1}}{r_{0}-a_{p_{0}-1}}, & 1 \leq j \leq p_{0}-2 .\end{cases}
$$

The (possible) critical values of $r_{0}$ are two. First

$$
\left(a_{0}, a_{1}, a_{2}, \ldots, a_{p_{0}-1}, r_{p_{0}}\right)=\left(a_{0}, a_{1}, a_{1}, \ldots, a_{1}, a_{0}\right)
$$

with

$$
\begin{aligned}
n_{1} & =\frac{\left(p_{0}-1\right)\left(p_{0}-2\right)}{2} a_{1}^{2}+2\left(p_{0}-1\right) a_{0} a_{1}+a_{0}^{2}, \\
n_{p_{0}} & =a_{0}^{2}
\end{aligned}
$$

whose positive solutions are $a_{0}=\sqrt{n_{p_{0}}}$ and

$$
a_{1}=\frac{\sqrt{2\left(p_{0}-1\right)\left(\left(p_{0}-2\right) n_{1}+p_{0} n_{p_{0}}\right)}-2\left(p_{0}-1\right) \sqrt{n_{p_{0}}}}{\left(p_{0}-1\right)\left(p_{0}-2\right)} .
$$

This yields

$$
r_{0}=\sqrt{\frac{2\left(p_{0}-1\right)}{p_{0}-2} n_{1}+\frac{2 p_{0}\left(p_{0}-1\right)}{\left(p_{0}-2\right)^{2}} n_{p_{0}}}-\frac{2}{p_{0}-2} \sqrt{n_{p_{0}}} .
$$

This critical value always exists. The second case is

$$
\left(a_{0}, a_{1}, a_{2}, \ldots, a_{p_{0}-1}, r_{p_{0}}\right)=\left(a_{0}, a_{1}, a_{1}, \ldots, a_{1}, a_{1}-a_{0}\right)
$$

with

$$
\begin{aligned}
n_{1} & =\frac{p_{0}\left(p_{0}-1\right)}{2} a_{1}^{2}+a_{0} a_{1}-a_{0}^{2}, \\
n_{p_{0}} & =n_{1}-\frac{p_{0}\left(p_{0}-1\right)}{2} a_{1}^{2} ;
\end{aligned}
$$

whose positive solutions are $a_{1}=\sqrt{\frac{2\left(n_{1}-n_{\left.p_{0}\right)}\right.}{p_{0}\left(p_{0}-1\right)}}$ and

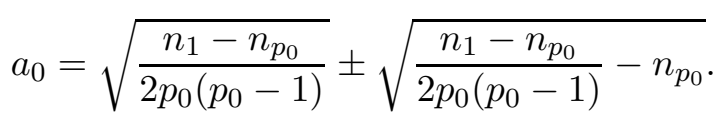

These \pm critical values exist if and only if

$$
n_{1} \geq\left(\left(p_{0}-1\right)^{2}+p_{0}^{2}\right) n_{p_{0}}
$$

and either of them yields

$$
r_{0}=\sqrt{\frac{2 p_{0}}{p_{0}-1}\left(n_{1}-n_{p_{0}}\right)} .
$$

If condition (5.7) holds, then the value of (5.8) is a local minimum (and the value of (5.6) is a local maximum). If condition (5.7) does not hold, then the value of (5.6) is a local minimum.

Arguing as we did with in first simplification we conclude that the value of (5.8), if (5.7) holds, and the value of (5.6), if (5.7) does not hold, is a global minimum. 


\section{REFERENCES}

[ARo] A. Alvarez, N. Rojas, Faithful representation of 2-step nilradicals of parabolic subalgebras of $A_{n}$, in progress.

[Be] Y. Benoist, Une Nilvariete Non Affine, J. Diff. Geom., Vol. 41,(1995), 21-52.

[Bi] G. Birkhoff, Representability of Lie algebras and Lie groups by matrices, Ann. of Math. (2), Vol. 38, (1937), 526-532.

[BoG] J. Bourgain, A. Gamburd, Uniform expansion bounds for Cayley graphs of $S L_{2}\left(F_{p}\right)$. Ann. of Math. (2), 167(2008), 625-642.

[B] D. Burde, Left-symmetric algebras, or pre-Lie algebras in geometry and physics, Central European J. of Math. 4(3), 323-357 (2006).

[BEdG] D. Burde, B. Eick, A. de Graaf, Computing faithful representations for nilpotent Lie algebras, J. of Alg.,Vol. 322, No. 3, (2009), 602-612.

[BM1] D. Burde, W. Moens, Minimal Faithful Representations of Reductive Lie Algebras, Archiv der Mathematik., Vol. 89, No. 6, (2007), 513-523.

[BM2] D. Burde, W. Moens, Faithful Lie algebra modules and quotients of the universal enveloping algebra, J. of Alg.,Vol. 325, No. 1, (2011), 440-460.

[CRo] L. Cagliero, N. Rojas, Faithful representation of minimal dimension of current Heisenberg Lie algebras, Int. J. Math. Vol. 20 (11), (2009), 1347-1362.

[dGN] W. de Graaf, W. Nickel, Constructing faithful representations of finitely-generated torsion-free nilpotent groups, J. Symbolic Comput.,Vol. 33, No. 1, (2002), 31-41.

[dG] W. de Graaf, Constructing faithful matrix representations of Lie algebras, Proceedings of the 1997 International Symposium on Symbolic and Algebraic Computation, ISSAC97, ACM Press, New York, 1997, 54-59.

[GSe] F. Grunewald, D. Segal, Some General Algorithms. I: Arithmetic Groups, Ann. of Math., Second Series, Vol. 112(1980), 531-583.

[J] N. Jacobson, Lie Algebras, Interscience Publishers, New York (1962).

$[\mathrm{K}] \quad$ H. Kim, Complete left-invariant affine structures on nilpotent Lie groups, J. Dif. Geometry 24(1986), 373-394.

[Mi] J. Milnor, On fundamental groups of complete affinely flat manifolds, Adv. Math., 25 (1977), 178-187.

[Ne] Y. Neretin, A construction of finite-dimensional faithful representation of Lie algebra, Rend. Circ. Mat. Palermo (2) Suppl. 71(2003), 159-161.

[N] W. Nickel, Matrix representations for torsion-free nilpotent groups by Deep Thought, J. of Algebra, 300(2006), 376-383.

[Ro] N. Rojas. Minimal Faithful Representation of the Heisenberg Lie algebra with abelian factor, Journal of Lie Theory 23 (2013), No. 4, 1105-1114.

[SX] P. Sarnak, X. Xue, Bounds for multiplicities of automorphic representations, Duke Math. J., 64(1991), 207-227.

[S] I. Schur. Zur Theorie vertauschbarer Matrizen, J. Reine Angew. Mathematik, 130 (1905), 66-76.

[Se1] D. Segal, Polycyclic Groups, Cambridge University Press.

[Se2] D. Segal, Free left-symmetric algebras and an analogue of the Poincaré-BirkhoffWitt Theorem, J. Algebra 164 (1994), 750-772.

COnicet - FamaF, Universidad Nacional de Córdoba, Argentina

E-mail address: cagliero@famaf.unc.edu.ar

Ciem, FCEFyn Universidad Nacional de Córdoba, Argentina

E-mail address: nrojas@efn.uncor.edu 